\title{
Differential regulation of caffeine metabolism in Coffea arabica (Arabica) and Coffea canephora (Robusta)
}

\author{
Charlène Perrois $\cdot$ Susan R. Strickler $\cdot$ Guillaume Mathieu • \\ Maud Lepelley • Lucie Bedon • Stéphane Michaux • \\ Jwanro Husson • Lukas Mueller • Isabelle Privat
}

Received: 4 June 2014/ Accepted: 8 September 2014/Published online: 24 September 2014

(c) The Author(s) 2014. This article is published with open access at Springerlink.com

\begin{abstract}
Caffeine is a metabolite of great economic importance, especially in coffee, where it influences the sensorial and physiological impacts of the beverage. Caffeine metabolism in the Coffea species begins with the degradation of purine nucleotides through three specific $\mathrm{N}$ methyltransferases: XMT, MXMT and DXMT. A comparative analysis was performed to clarify the molecular reasons behind differences in caffeine accumulation in two Coffea species, namely Coffea arabica and Coffea canephora var. robusta. Three different genes encoding $\mathrm{N}$-methyltransferase were amplified in the doubled haploid Coffea canephora: CcXMT1, CcMXMT1 and CcDXMT. Six genes were amplified in the haploid Coffea arabica: CaXMT1, CaXMT2, CaMXMT1, CaMXMT2, CaDXMT1, and CaDXMT2. A complete phylogenic analysis was performed to identify specific key amino acids defining enzymatic function for each protein identified. Furthermore, a quantitative gene-expression analysis was conducted on leaves and on maturing coffee beans, simultaneously analyzing caffeine content. In the different
\end{abstract}

Electronic supplementary material The online version of this article (doi:10.1007/s00425-014-2170-7) contains supplementary material, which is available to authorized users.

C. Perrois · G. Mathieu - M. Lepelley - L. Bedon ·

S. Michaux $\cdot$ J. Husson $\cdot$ I. Privat $(\bowtie)$

Nestlé R\&D Center, 101 Av. Gustave Eiffel, Notre Dame D’Oé,

BP 49716, 37097 Tours, France

e-mail: isabelle.privat@rdto.nestle.com

S. R. Strickler · L. Mueller

Boyce Thompson Institute for Plant Research, Tower Road, Ithaca, NY 14853, USA varieties analyzed, caffeine accumulation is higher in leaves than in the coffee bean maturation period, higher in Robusta than in Arabica. In Robusta, CcXMT1 and CcDXMT gene expressions are predominant and transcriptional activity is higher in leaves than in maturing beans, and is highly correlated to caffeine accumulation. In Arabica, the CaXMT1 expression level is high in leaves and CaDXMT2 as well to a lesser extent, while global transcriptional activity is weak during bean maturation, suggesting that the transcriptional control of caffeine-related genes differs within different organs and between Arabica and Robusta. These findings indicate that caffeine accumulation in Coffea species has been modulated by a combination of differential transcriptional regulation and genome evolution.

Keywords Arabica - Beans - Caffeine - Expression . Leaves $\cdot N$-methyltransferase $\cdot$ Robusta

\begin{tabular}{|c|c|}
\hline \multicolumn{2}{|c|}{ Abbreviations } \\
\hline $\mathrm{Ca}$ & Coffea arabica \\
\hline$C c$ & Coffea canephora var. robusta \\
\hline Arabica & Coffea arabica \\
\hline Robusta & Coffea canephora var. robusta \\
\hline rpl39 & Large ribosomal subunit 39 protein \\
\hline UBQ & Ubiquitin protein \\
\hline q-RT-PCR & $\begin{array}{l}\text { Quantitative real-time polymerase chain } \\
\text { reaction }\end{array}$ \\
\hline gDNA & Genomic DNA \\
\hline XMT & Xanthosine- $N$-methyltranferase \\
\hline MXMT & $\begin{array}{l}\text { 7-methylxanthine- } N \text {-methyltransferase or } \\
\text { theobromine synthase }\end{array}$ \\
\hline DXMT & $\begin{array}{l}\text { 3,7-dimethylxanthine- } N \text {-methyltranferase or } \\
\text { caffeine synthase }\end{array}$ \\
\hline MTL & Methyltransferase-like protein \\
\hline
\end{tabular}




\section{Introduction}

Coffee is an important crop with more than seven million tons of green beans produced every year. After oil, coffee ranks second in international trade exchanges. The two main species cultivated throughout the tropical world are Coffea arabica and Coffea canephora, which represent 70 and $30 \%$, respectively in world production. In terms of cup quality, Coffea arabica (Arabica) is appreciated by consumers due to better flavor and high acidity compared with Coffea canephora (Robusta), famous for its bitterness and intense dark flavor. Coffee quality, with complex variables, depends significantly on the fluctuating biochemical composition of the bean during fruit maturation (Simkin et al. 2006; Lepelley et al. 2007; Privat et al. 2008; Salmona et al. 2008) as well as control on the genetic level (Montagnon et al. 1998; Leroy et al. 2006). The principal molecules accumulated in coffee beans are caffeine, chlorogenic acids, lipids, sucrose, fat, and proteins. These different aroma precursors are transformed during roasting through Maillard reactions. Typically, chlorogenic acids and caffeine are responsible for coffee bitterness, while free carbohydrates like sucrose generate flavor compounds by interacting with amino acids. Furthermore, sucrose lends sweetness to the final beverage taste. Among the compounds present in coffee beans, caffeine is among the best known and most commonly studied because of its physiological effects on humans and its role in coffee plant resistance.

Caffeine is a clear target for breeding programs to obtain natural low-caffeine-content coffee or on the contrary to increase caffeine content to produce darker and stronger coffee. Caffeine accumulation studies among the Coffea species (Ky et al. 2001; Campa et al. 2004, 2005; Ashihara and Suzuki 2004) have highlighted a broad range of diversity from no caffeine in $C$. pseudozanguebariae to the highest content in Coffea canephora (Robusta). A segregation study on an interspecific cross between Coffea liberica and Coffee pseudozanguebariae showed two major QTLs controlling caffeine and chlorogenic-acid content in beans (Ky et al. 2013). Breeding strategies, including QTL identification combined with candidate-gene validation, are highly valuable and may help identify the caffeine-metabolic pathway in Coffea species characterized by different levels of caffeine content.

The biosynthetic pathway of caffeine has been intensively studied and it has now been established that caffeine is synthesized through the sequential three-step methylation of xanthosine derivatives at positions 7-N, 3-N and $1-\mathrm{N}$, with a nucleosidase reaction leading to the synthesis of the following molecules: the 7-methylxanthosine, the 3,7-methylxanthine (theobromine) and last, the 1,3,7trimethylxanthine (caffeine) (Ashihara and Crozier 1999; Ashihara and Suzuki 2004). The three N-methyltransferases were designated as xanthosinemethyltransferase (XMT), 7-methylxanthine transferase (MXMT) and 3,7dimethylxanthine methyltransferase (DXMT). Numerous studies have been launched aiming to identify the genes involved in caffeine biosynthesis (Ogawa et al. 2001; Uefuji et al. 2003; Mizuno et al. 2003b). The different published sequences exhibit a high degree of sequence similarity $(>80 \%)$ with each other. They also show substrate specificity allowing us to define key motifs/amino acids involved in enzymatic activity (Mizuno et al. 2003a; Uefuji et al. 2005; McCarthy et al. 2007). Most of the available sequences have been identified in Coffea arabica but before this year, no comparative analysis had been performed to elucidate the molecular reasons for the difference in caffeine accumulation in different Coffea species. Our major focus was to perform a comparative analysis at different levels: (1) gene identification and annotation, (2) caffeine quantification and, (3) analysis of the gene expression encoding the different $N$-methyltransferases in Coffea species characterized by varying caffeine contents. The main objective is to propose a first explanation for the differences in caffeine accumulation in coffee.

\section{Materials and methods}

Plant material fresh cherries were harvested from coffee trees cultivated in Quito, Ecuador, at different stages of development [small green fruit (SG), large green fruit (LG), yellow fruit (Y) and red fruit (R)] (Privat et al. 2008). For each maturation stage, only 15-20 coffee cherries were available for each genotype analyzed. Therefore, performing three biological replicates on the same genotype was not possible. Thus, to draw conclusions for each species, several genotypes were used that were considered as biological replicates. Four genotypes were used for this study: Coffea arabica L. cv. Caturra (Arabica) CCCA12 and CCCA02, and Coffea canephora var. robusta (Robusta) FRT05 and FRT64. Due to the fact that Robusta cherries develop over a period of 9-11 months while Arabica fruits develop over a 6-8 month period (Wintgens 2004), the ripening stages were classified by the relative parameters of size, weight and color change, rather than by weeks after flowering (Bargel and Neinhuis 2005). Fresh tissues were frozen immediately and then packaged in dry ice or frozen to $-25{ }^{\circ} \mathrm{C}$ for transportation, then stored at $-80{ }^{\circ} \mathrm{C}$ until use. The bean and pericarp tissues were separated for each 
stage of maturation. Young and mature leaves were collected from different genotypes grown under greenhouse conditions in Tours, France: Coffea arabica L. $c v$. Caturra (Arabica) CCCA12, CCCA02, CCCA24, CCCA18; Coffea canephora var. robusta (Robusta) FRT05, FRT64. Last, leaves from the Coffea canephora accession DH200-94 and the Coffea arabica ET39-DH3 were collected to characterize the genes or cDNA sequences encoding the caffeine-synthesis-related genes. Frozen tissues were then homogenized using a SPEX CertiPrep 6800 freezer mill with liquid nitrogen. These different samples were used for RNA, genomic extraction and/or biochemical analysis as previously (Lepelley et al. 2007; Privat et al. 2008).

\section{Extraction of total RNA and cDNA preparation}

Samples stored at $-80{ }^{\circ} \mathrm{C}$ were ground into a powder and total RNA was extracted from this powder using the method described previously (Rogers et al. 1999). Samples were treated with DNase using the "Qiagen RNase-Free DNase" kit in accordance with the manufacturer's instructions on removing DNA contamination. All RNA samples were analyzed by formaldehyde agarose gel electrophoresis and by visual inspection of the ribosomal RNA bands on ethidium bromide staining. Furthermore, the concentration was determined on a Nanodrop spectrophotometer (NanoDrop Technologies). Using oligo ( $\mathrm{dT}_{20}$ ) as a primer, cDNA was prepared from $1 \mu \mathrm{g}$ total RNA, according to the protocol in the superscript III reverse transcriptase kit (Invitrogen, Carlsbad, CA, USA). The first-strand cDNA synthesis incubation step was performed for $50 \mathrm{~min}$ at $50{ }^{\circ} \mathrm{C}$. The cDNA samples were then diluted 100 -fold in sterilized water and stored at $-20{ }^{\circ} \mathrm{C}$ for later use in a quantitative real-time polymerase chain-reaction (q-RT-PCR) analysis or cDNA full-length amplification.
cDNA and genomic amplification for caffeine-related gene characterization

To amplify full-length cDNA or genomic sequences encoding the different $N$-methyltransferases involved in caffeine synthesis, the following primer sets-CAF1-ATG $\left({ }^{5^{\prime}}\right.$ ATGGAGCTCCAAGAAGTCCTGCA $\left.{ }^{3{ }^{\prime}}\right)$, CAF1-STOP $\left({ }^{5^{\prime}}\right.$ TTACACGTCTGACTTCTCTGGCT $\left.{ }^{3^{\prime}}\right)$ and CAF2-ATG $\left({ }^{5^{\prime}}\right.$ ATGGAGCTCCAAGAAGTCCTGCG $\left.{ }^{3 \prime}\right)$, CAF2-STOP $\left({ }^{5^{\prime}}\right.$ TTACATGTCTGACTTCTCTGGCT $\left.{ }^{3^{\prime}}\right)$ were designed on the consensus sequence obtained from the alignment of the different $\mathrm{N}$-methyltransferases available in the public databases. These two primer sets were used to perform PCR reactions using cDNA or genomic DNA samples prepared from Coffea canephora accession DH-200-94 and the Coffea arabica ET39-DH3. The PCR reactions were performed in $50 \mu \mathrm{L}$ reactions as follows: $5 \mu \mathrm{L}$ of cDNA or gDNA; $1 \times$ buffer, $800 \mathrm{nM}$ of each gene-specific primer, $200 \mu \mathrm{M}$ of each dNTP, and $0.5 \mathrm{U}$ of LA Taq polymerase (Invitrogen). After denaturing at $94{ }^{\circ} \mathrm{C}$ for $5 \mathrm{~min}$, the amplification consisted of 35 cycles of $1 \mathrm{~min}$ at $94{ }^{\circ} \mathrm{C}$, $1 \mathrm{~min}$ at $55^{\circ} \mathrm{C}$ and $2 \mathrm{~min}$ at $72{ }^{\circ} \mathrm{C}$. An additional final step of elongation was carried out at $72{ }^{\circ} \mathrm{C}$ for $7 \mathrm{~min}$. Fragments obtained were purified from agarose gel, cloned and sequenced. To prevent any errors from PCR amplification, each PCR was performed in duplicate, the fragments being cloned separately from two independent ligations.

\section{DNA Sequencing and sequences analysis}

For DNA sequencing, recombinant plasmid DNA was prepared and then sequenced by GATC (Konstanz, Germany). Computer analysis was performed using DNA Star (Lasergene, DNAstar Inc., Madison, WI, USA) software. Sequence homologies were verified against GenBank databases using BLAST programs (Altschul et al. 1990). The sequence encoding each gene was validated whenever the

Table 1 Identification of the genes encoding the $N$-methyltransferase involved in caffeine synthesis in Coffea arabica and Coffea canephora species

\begin{tabular}{|c|c|c|c|c|c|}
\hline \multirow{2}{*}{$\frac{\text { Species }}{\text { Coffea canephora } \text { DH-200-94 }}$} & \multirow{2}{*}{$\frac{\text { Gene name }}{\text { CcXMT1 }}$} & \multicolumn{2}{|c|}{ Genomic sequence } & \multicolumn{2}{|c|}{ mRNA sequence } \\
\hline & & JX978509 & $1994 \mathrm{pb}$ & JX978518 & $1119 \mathrm{~b}$ \\
\hline & CсMXMT1 & JX978507 & $1829 \mathrm{pb}$ & JX978517 & $1137 \mathrm{~b}$ \\
\hline & $C c D X M T$ & JX978506 & $2006 \mathrm{pb}$ & JX978516 & $1155 \mathrm{~b}$ \\
\hline \multirow[t]{6}{*}{ Coffea arabica ET39-DH3 } & CaXMT1 & JX978514 & $1987 \mathrm{pb}$ & JX978521 & $1119 \mathrm{~b}$ \\
\hline & CaXMT2 & JX978515 & $2038 \mathrm{pb}$ & JX978522 & $1158 \mathrm{~b}$ \\
\hline & CaMXMT1 & JX978511 & $1838 \mathrm{pb}$ & JX978519 & $1137 \mathrm{~b}$ \\
\hline & СаMXMT2 & JX978512 & $2010 \mathrm{pb}$ & JX978520 & $1155 \mathrm{~b}$ \\
\hline & CaDXMT1 & JX978510 & $2063 \mathrm{pb}$ & KF678863 & $1155 \mathrm{~b}$ \\
\hline & CaDXMT2 & KJ577792 & $2006 \mathrm{pb}$ & KJ577793 & $1155 \mathrm{~b}$ \\
\hline
\end{tabular}

For each species, the amplified genomic sequences as well as the corresponding mRNA have been annotated. For each sequence, the accession number in NCBI is specified as well as the sequence length 
same sequence was obtained from two independent PCRs. Each sequence identified in this article was submitted to the NCBI database (Table 1).

\section{Quantitative real time PCR}

Alignment of the genomic sequences encoding the caffeine-related genes (Supplementary Fig. S1) shows a high similarity between the different sequences identified. The Exon 3 region shows the highest level of polymorphism within the different genes, leading us to design all the primers/TaqMan probe pairs in this region. For each gene, primers/probe pairs were designed on the different sequences available using PRIMER EXPRESS software (Applied Biosystems, Foster City, CA, USA) (Supplementary Table S1). The TaqMan PCR reactions were carried out according to the manufacturer's instructions (Applied Biosystems). For all primers/probe sets, standard curves were generated using serial dilutions of plasmid DNA containing the appropriate target-gene sequence. $\mathrm{Ct}$ values were determined and plotted versus the natural logarithm of the DNA concentration. Regression analysis provided a linear function from which the PCR efficiency could be calculated using the equation $E=e^{-1 / m}-1$, where $E$ is the PCR efficiency, $e$ Euler's number and $m$ the slope of the regression function. Efficiency values were determined for each gene. Furthermore, to prevent any unspecific amplification, the primer/probe designs for one specific gene were tested on the different sequences identified in this publication. Only the primers/probe pairs that showed no unspecific amplification were used for further analysis. The cDNA samples used in this experiment have been described above. All reactions contained $1 \times$ TaqMan buffer (Applied Biosystems) and $5 \mathrm{mM} \mathrm{MgCl} 2,200 \mu \mathrm{M}$ each of dATP, dCTP, dGTP and dTTP, 100-fold dilution of cDNA corresponding to $0.001 \mu \mathrm{g}$ of original RNA and 0.625 units of AmpliTaq Gold polymerase. A PCR was carried out using $800 \mathrm{nM}$ of each gene-specific primer, forward and reverse, and $200 \mathrm{nM}$ of the corresponding TaqMan probe. Reaction mixtures were incubated for $2 \mathrm{~min}$ at $50{ }^{\circ} \mathrm{C}, 10 \mathrm{~min}$ at $95{ }^{\circ} \mathrm{C}$, followed by 40 amplification cycles of $15 \mathrm{~s}$ at $95{ }^{\circ} \mathrm{C} / 1 \mathrm{~min}$ at $60{ }^{\circ} \mathrm{C}$. Samples were quantified in the GeneAmp 7500 Sequence Detection System (Applied Biosystems). Transcript levels were normalized using rpl39 (large ribosomal subunit 39) and $U B Q$ (ubiquitin-like protein) as reference genes (Cruz et al. 2009; Lepelley et al. 2012). The values represent the mean of three technical repetitions \pm standard deviation.

\section{Caffeine quantification}

The coffee material was ground with liquid nitrogen, sifted through a $500 \mu \mathrm{m}$ sieve, and immediately stored at $-20{ }^{\circ} \mathrm{C}$ until use. The ground coffee material $(10 \mathrm{mg})$ was extracted in de-ionized water containing $70 \%$ methanol. The resulting mixture was macerated by stirring for $30 \mathrm{~min}$ at $40{ }^{\circ} \mathrm{C}$. The mixture was then filtered using GHP Acrodisc $0.2 \mu \mathrm{m}$ filter. The alkaloids and chlorogenic acids were analyzed by HPLC (U3000 from Dionex) on an ACE RP18 $(250 \times 4 \mathrm{~mm}, 5 \mu \mathrm{m})$ column. The injected sample volume was $10 \mu \mathrm{L}$ with a flow rate of $0.8 \mathrm{~mL} / \mathrm{min}$. The caffeine content is expressed in percentage of dry weight (\% DW).

\section{Bioinformatics and phylogenic analysis}

Protein alignments were generated using Clustal W (Thompson et al. 1994) with default values as implemented in Mega v. 5.2 (Tamura et al. 2011). A visual inspection of the alignment was conducted to ensure optimal results. Phylogenetic analysis was performed using 371 amino acid sites including gaps and 19 sequences. A maximum likelihood tree was generated with Mega v. 5.2 (Tamura et al. 2011) using a Poisson substitution model and gamma-rate distribution among sites. Nearest-neighbor-interchange was used as the heuristic tree search method with 100 bootstrap samples. Jalview v. 2.0 (Waterhouse et al. 2009) was implemented to produce an alignment image for publication. A tree image for publication was generated using FigTree v 1.4 (http://tree.bio.ed.ac.uk/software/figtree/). To find putative amino acids conferring functional specificity to each of the three $N$-methyltransferase clusters, the alignment was imported into multi-Harmony (Brandt et al. 2010) along with the protein structure of the $N$-methyltransferase 2EG5E from $C$. canephora as a reference.

\section{Results}

Isolation and characterization of caffeine metabolismrelated genes

To identify the different genes involved in the final steps of the caffeine biosynthesis pathway in two Coffea species characterized by different levels of caffeine content, we decided to make use of the fact that previous sequences shared high similarity (Ogawa et al. 2001; Uefuji et al. 2003; Mizuno et al. 2003b; McCarthy and McCarthy 2007) and to design "universal" caffeine gene primers (see Materials and methods). To eliminate the natural polymorphism/allelism existing in Coffea species that could interefere with sequence annotation, we selected two specific Coffeas: the doubled-haploid Coffea canephora Robusta DH-200-94, diploïd but strictly homozygous, and the haploid Coffea arabica ET39-DH3 which has only one copy of each subgenome. For the two species, the different 
genomic sequences characterized are listed in Table 1 . The cDNA sequences were amplified from mRNA extracted from leaf samples. When possible, the genomic and mRNA sequences were aligned, successfully indicating the intron/ exon junction and identifying the correlation between genomic and mRNA sequences. The alignment between all the genomic sequences encoding $N$-methyltransferases which are involved in caffeine metabolism shows a similar organization (Supplementary Fig. S1). The genomic sequence length varies from 1,829 to 2,063 bp. Each gene has four exons and three introns. Three different genes encoding $N$-methytransferase were amplified in the doubled haploid Coffea canephora CcXMT1 (JX978509), CcMXMT1 (JX978507), CcDXMT (JX978506). Six genes were amplified in the haploid genome of Coffea arabica, i.e., CaXMT1 (JX978514), CaXMT2 (JX978515), CaMXMT1 (JX978511), CaMXMT2 (JX978512), CaDXMT1 (JX978510) and CaDXMT2 (KJ577792). Interestingly, for each enzyme, two paralogue genes were identified in the Coffea arabica genome (Table 1). For each gene, the corresponding cDNA was amplified using RNA extracted from leaves (Table 1). The highest variability between the coding sequences is clearly localized in Exon 4 (Supplementary Fig. S1), where specific gaps in the sequence differentiate XMT1 from the MXMT1 genes in Arabica and Robusta. Furthermore, variability, especially in the Intron 2 and Intron 3 regions, helped to identify the specific orthologues between the two Coffea species. The protein sequences deduced from each identified gene were aligned (Fig. 1). A phylogenic analysis was performed including the 9 proteins identified in this article as well as various $N$ methyltransferases previously published for different Arabica and Robusta genotypes (Fig. 2). This phylogenic analysis indicated that the different $\mathrm{N}$-methyltransferases involved in caffeine synthesis belong to 3 different clusters which align with the function of each protein. Indeed, Clusters I, II and III correspond respectively to XMT, MXMT and DXMT. The different proteins were shown to share more than $80 \%$ similarity throughout the two Coffea species. It is interesting to note that within Clusters I, II and III, the percentage of similarity is between 94 and $100 \%$. The first cluster includes the different genes encoding the XMT enzyme. The two genes CaXMT1 (JX978521) and CcXMT1 (JX978518) were identified (Fig. 2) and are closely related to proteins published in the databases CaXMT1 (AB048793) (Ogawa et al. 2001) and CcXMT1 (A4GE69) (McCarthy and McCarthy 2007; McCarthy et al. 2007). An additional gene, CaXMT2 (JX978522), was identified in the haploid Coffea arabica that has no closely related gene in the Coffea canephora genome. These results suggest that in Coffea arabica, the genes CaXMT1 and CaXMT2 are, respectively encoded by the Robusta and Eugenioides subgenomes. The second cluster includes the genes encoding the MXMT enzyme. The genes CaMXMT1 (JX978519) and CCMXMT1 (JX978517) encode the same protein, homologous to the CaMXMT1 (AB048794) (Ogawa et al. 2001). As it has already been observed for CaXMT2, a second gene CaMXMT2 (JX978520) was also identified (Table 1; Fig. 2); it is clearly the same protein as CTS2 (AB054841) (Mizuno et al. 2003b), also identified in Coffea arabica. This gene has no homologue in Coffea canephora. In addition, MXMT1 and MXMT2 proteins share $94 \%$ similarity. The third cluster includes the genes encoding the DXMT enzyme. In Coffea arabica, two genes, CaDXMT1 (KF678863) and CaDXMT2 (KJ577793), have been identified while in Coffea canephora, only one gene, CcDXMT (JX978516), was identified. Their predicted amino acids share $94 \%$ identity to each other. The members of Cluster III (Fig. 2) define two sub-clusters depending on the origin of the sequence (Arabica or Robusta), suggesting variability among the protein sequences according to the two Coffea species analyzed. The proteins CaDXMT2 and CcDXMT are highly homologous and correspond to the protein CcDXMT (A4GE70) and CCS1 (AB086414) (Mizuno et al. 2003a) previously identified. CaDXMT1 (KF678863) corresponds to CaDXMT1 (AB084125) (Uefuji et al. 2003) and CtCS7 (Uefuji et al. 2003). CaDXMT2 and CaDXMT1 proteins are, respectively encoded by the Robusta and Eugenioides sub-genomes in Coffea arabica genome. To find putative amino acids conferring functional specificity to each of the three clusters, the alignment (Fig. 1) was imported into Multi-Harmony software (Brandt et al. 2010) along with the protein structure of $C$. canephora XMT (2EG5E as a reference). The result of this analysis has highlighted 16 amino acids capable of defining the signature for the members of each cluster (Table 2). Three amino acids are unique for one of the defining clusters. Identifying these residues significantly clarifies the different protein characteristics and will aid in annotating future $\mathrm{N}$-methyltransferases in Coffea species.

\section{Caffeine and theobromine accumulation}

Biochemical analysis indicated an accumulation of theobromine and caffeine in the leaves at both stages in the C. canephora and C. arabica genotypes (Fig. 3a). Young leaves contain the highest concentration of caffeine in Robusta and Arabica genotypes. On average, Robusta accumulates $3 \%$ (expressed as the percentage of dry weight) and Arabica $1.6 \%$ (DW) of caffeine in young leaves. For both species, in mature leaves, caffeine content drops to $70 \%$ of the initial quantified caffeine content in young leaves. Theobromine is accumulated less in young leaves than caffeine in both species. In young leaves, Robusta accumulates $1.4 \%$ (DW) of theobromine, while 
Fig. 1 Multiple sequence alignment of nineteen $\mathrm{N}$ methyltransferases involved in caffeine metabolism with multiHarmony results. The Seq-Harm track displays the Sequence Harmony score for each site ranging from 0 to 1 . The Multirelief track shows the weight for each site. GenBank accession numbers are as follows: CaXMT1 (AB048793); CcXMT1 (A4GE69); CcXMT1* (JX978518); CaXMT1* (JX978521); CaXMT2* (JX978522); CcMXMT1* (JX978517); CaMXMT1 (AB048794); CaMXMT1* (JX978519); CaMXMT2 (AB084126); CaMXMT2* (JX978520); CTS1 (AB034700); CTS2 (AB054841); CaDXMT1* (KF678863); CcDXMT* (JX978516); CaDXMT1 (AB084125); CtCS7 (AB086415); CCS1 (AB086414); CcDXMT (A4GE70); CaDXMT2* (KJ577793). The sequences marked with an asterisk have been identified in this article

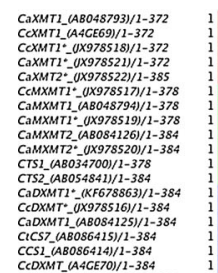

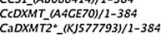

mutti-elelief

1- Sea-Harm

Conservation Quality

Consensus

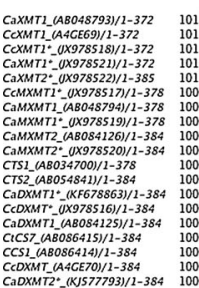

multi-Reli

- Seq-Harm

Conservation

Quality

Consensus

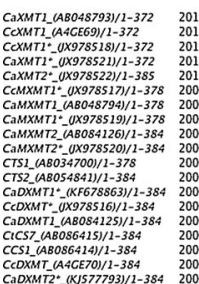

multi-Relief

1- Sea-Harm

Conservation

Quality

Consensus

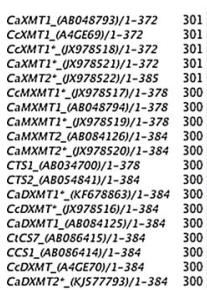

multi-Relief

1- Seq-Harm

Conservation

Quality

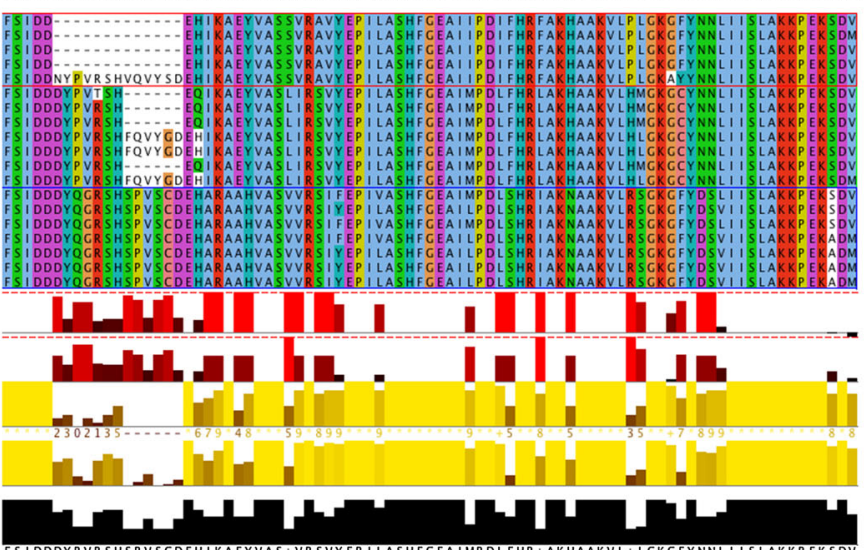

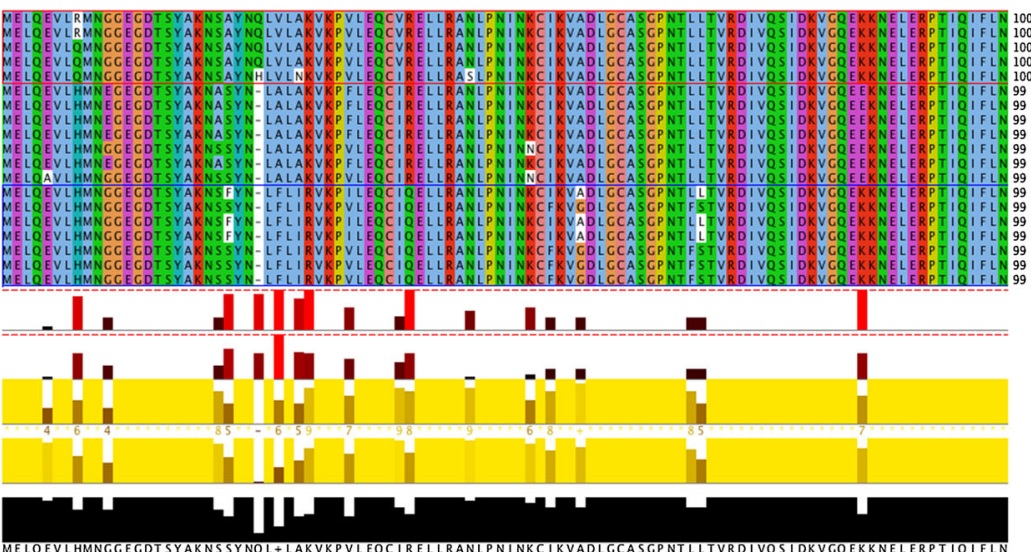
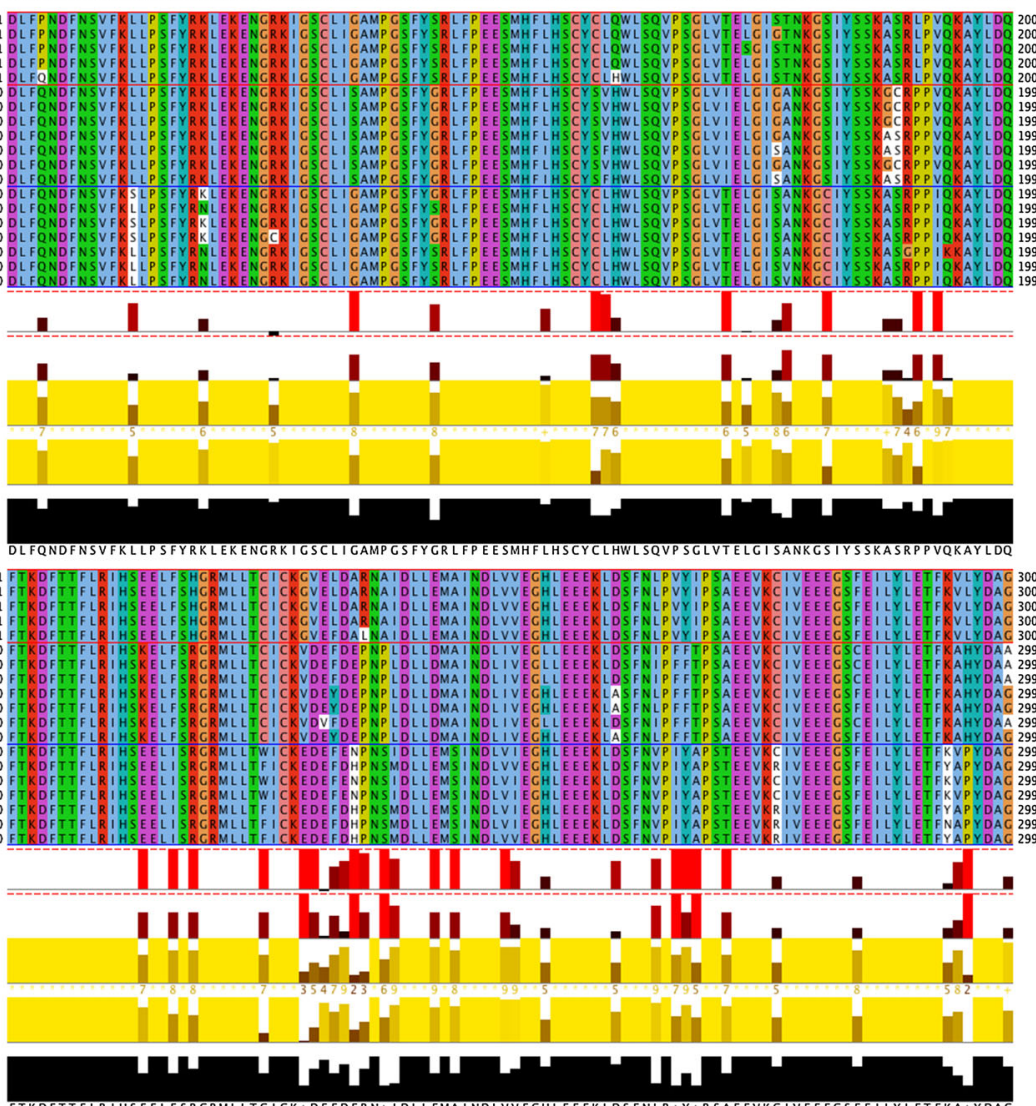

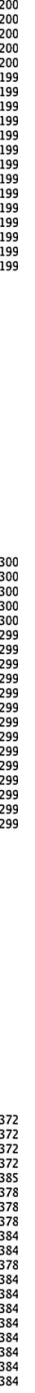




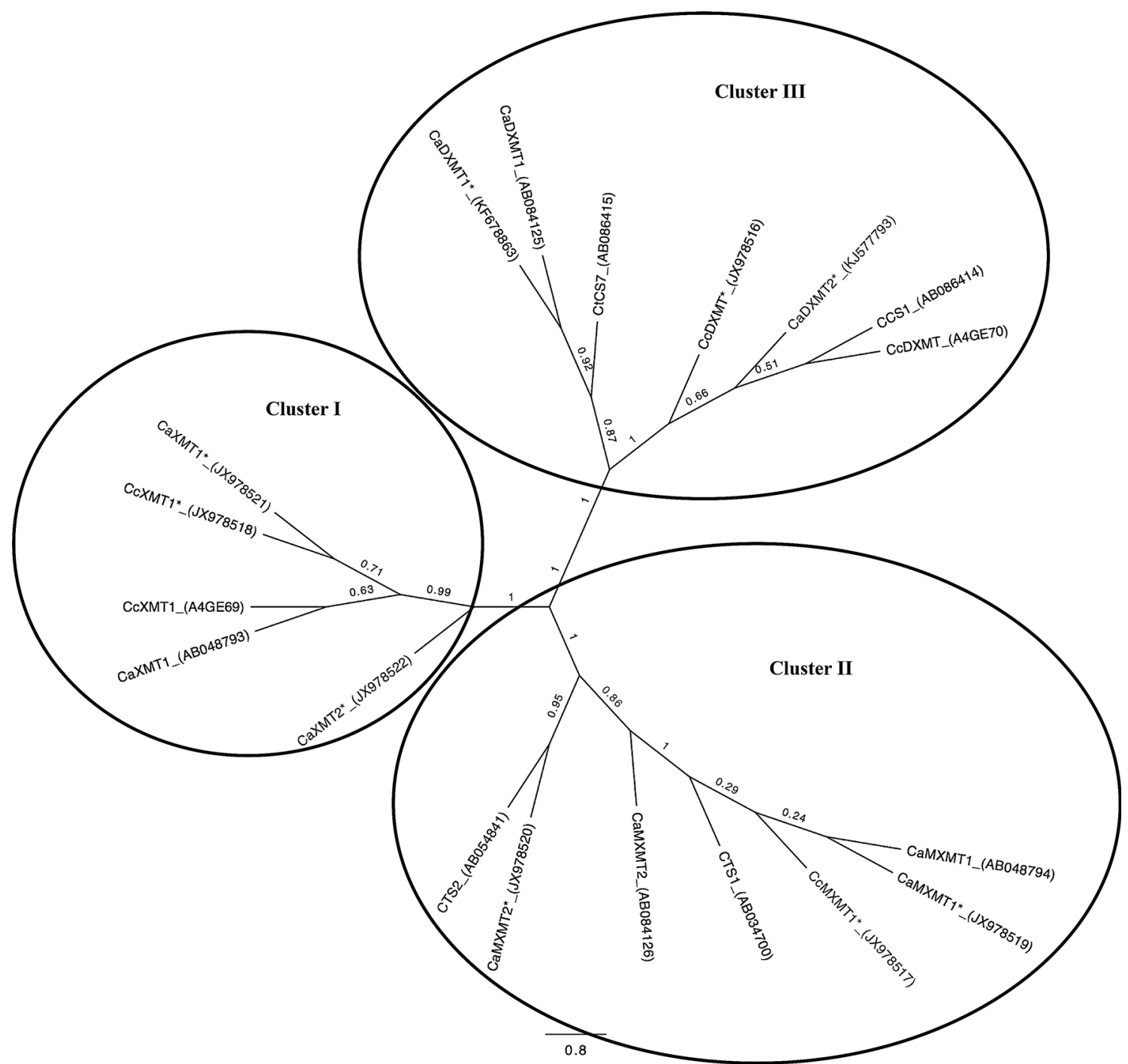

Fig. 2 Unrooted maximum likelihood tree based on the alignment of $19 \mathrm{~N}$-methyltransferases involved in caffeine metabolism. GenBank accession numbers are as follows: CaXMT1 (AB048793); CcXMT1 (A4GE69); CcXMT1* (JX978518); CaXMT1* (JX978521); CaXMT2* $^{*} \quad$ (JX978522); CcMXMT1* (JX978517); CaMXMT1 (AB048794); CaMXMT1* (JX978519); CaMXMT2 (AB084126);

Arabica accumulates $50 \%$ less. The reduction of theobromine in mature leaves is particularly apparent for both species, especially for Arabica, where theobromine is no longer detected at all in mature leaves. Interestingly, theobromine is not detected in the beans of Arabica or Robusta during the maturation process (Fig. 3b). In the same samples, caffeine is clearly accumulated in both species, but its highest content is found in Robusta (twice as much) starting even at the earliest stage (SG). Nevertheless, the accumulation profile is the same for Arabica and Robusta. During the expansion phase (from SG to LG stages), caffeine is accumulated even more significantly for Arabica. From LG to YG stages, caffeine content decreases
CaMXMT2* (JX978520); CTS1 (AB034700); CTS2 (AB054841); CaDXMT1* (KF678863); CcDXMT* (JX978516); CaDXMT1 (AB084125); CtCS7 (AB086415); CCS1 (AB086414); CcDXMT (A4GE70); CaDXMT2* (KJ577793). The sequences marked with an asterisk have been identified in this article. Clusters I, II and III correspond to XMT, MXMT and DXMT protein, respectively

and stabilizes during the last two stages of maturation (YG and $\mathrm{RG}$ ). In the mature bean (RG stage), the caffeine content is $50 \%$ higher in Robusta than in Arabica. Surprisingly, comparing caffeine accumulation in leaves and beans reveals that caffeine biosynthesis is highest in young leaves.

Gene expression during leaf and fruit development

Gene expression analysis was performed on leaf and coffee bean development to identify the major differences in the expression pattern of the different genes involved in the final steps of caffeine metabolism. As the different genes 
Table 2 Amino acids that support the presence of the four clusters derived from the phylogenetic analysis (see Fig. 1) and multi-Harmony data mining (see Fig. 2)

Amino acids in

\begin{tabular}{llll}
\hline Position $^{\mathrm{a}}$ & Cluster I XMT & Cluster II MXMT & Cluster III DXMT \\
\hline 31 & K & K & R \\
159 & C & S & C \\
191 & L & P & P \\
193 & V & V & I \\
217 & F & F & I \\
230 & $G^{b}$ & V & E \\
235 & A & E & H/N \\
238 & A & $P$ & S \\
245 & A & A & S \\
268 & Y & F & Y \\
296 & $L$ & $H$ & $P$ \\
316 & S & L & V \\
341 & F & L & I \\
344 & H & H & N \\
350 & P & H & R \\
357 & N & N & D \\
\hline
\end{tabular}

a Positions according to the CaXMT1 (JX978518) protein sequence

b The amino acids which are highly indicative for the three clusters are italicized. These amino acids are unique for one of the defined clusters

are over $80 \%$ identical to each other at the nucleotide level, we decided to conduct a gene expression analysis using quantitative RT-PCR using a TaqMan probe. This technology allowed us to design highly specific probes/ primers for each gene. The design was carried out in the most variable region of the coding sequence, i.e., Exon 4 (Supplementary Fig. S1). The gene expression pattern is clearly different between Arabica and Robusta, with drastic divergence in young leaves (Fig. 4a, b). CcXMT1 and CcDXMT are highly expressed in the different Robusta genotypes, CcDXMT being higher than CcXMTI. CcMXMT1 is expressed at a lower level in FRT05 and FRT32 genotypes, while it is significantly expressed in FRT65. In the different Arabica genotypes, CaXMT1 transcripts show high accumulations in young leaves compared to what we observe in Robusta. The highest accumulation is observed in the CCCA24 genotype. $\mathrm{Ca}$ $X M T 2$, the second gene encoding XMT enzyme in Arabica is slightly expressed compared to CaXMTl and detected only in young leaves. CaMXMT1 is detected also only in young leaves at low level compared to CaXMT1 for example. CaMXMT1 and CcMXMT1 are expressed similarly in young leaves while both genes are undetectable in mature leaves. CaMXMT2 is expressed at very low level in young and mature leaves. The most remarkable difference in Arabica is that CaDXMT2 is almost undetectable in all the Arabica genotypes in young leaves while the corresponding gene $C c D X M T$ in Robusta is highly expressed in young leaves. In both Arabica and Robusta genotypes, the gene expression pattern is modified in mature leaves. Overall gene expression is largely reduced; $C c X M T 1$ and CaXMT1 are still detectable, at a higher level for CaXMT1 in the Arabica genotypes. On the contrary, while CcDXMT is still detected in the mature leaves in the Robusta genotypes, CaDXMT2 is barely detected in the different Arabica genotypes.

During coffee bean development, the expression profile is also quite different between the Arabica and Robusta genotypes (Fig. 4c, d). Overall gene expression is strongly reduced during bean maturation. When a specific transcript is detected, it is accumulated approximately 10 times less than during leaf development in Robusta and 70 times less in Arabica. In Robusta genotypes, as it has already been observed in the young leaf, $C c X M T 1$ and $C c D X M T$ are highly expressed at the same level in the bean expansion phase (SG and LG). Expression decreased drastically in the last stages of maturation, becoming almost undetectable in the RG stage. CcMXMT1 is only slightly expressed at all stages. For the different Arabica genotypes, all the detected genes (CcXMT1, CaMXMT1, CaMXMT2 and CaDXMT2) are accumulated at very low levels. CaDXMT2 accumulation is only slightly higher in the SG and LG stages.

\section{Discussion}

The sequences of the different N-methyltransferase genes involved in the biosynthesis of caffeine were previously identified in coffee and other plant species (Ashihara et al. 2008). However, the fact that these enzymes share highly conserved domains makes it difficult to identify genespecific sequences for each methyltransferase. Also, the previously published sequences were identified in Coffea arabica, allotetraploid species $(2 n=4 x=44$ chromosomes) which result from the natural hybridization of two diploid species: C. canephora (Robusta) and C. eugenioides (Lashermes et al. 1999). The inter- and intra-polymorphism observed between the two sub-genomes greatly hinders the process of identifying the different genes encoding the $N$-methyltransferases. To eliminate any natural polymorphism/allelism existing in the Coffea species that could interfere with sequence annotation, specific coffee plants were selected. First, the double-haploid Coffea canephora Robusta DH-200-94 was chosen, which is strictly homozygous for each locus. Each polymorphism corresponds to a different sequence and consequently to a different gene. Second, the haploid Coffea arabica ET39DH3, with only one copy of each sub-genome, was used to 
Fig. 3 Caffeine and theobromine content during leaf or grain development in different Coffea species. a Caffeine and theobromine were quantified during leaf development in young (YL) and mature leaves (ML) for different Coffea arabica genotypes (CCCA 02, CCCA18, CCCA24), Coffea canephora var. robusta genotypes (FRT05, FR32, FRT65). b Caffeine and theobromine were quantified during coffee bean maturation for different Coffea arabica genotypes (CCCA 02, CCCA18, CCCA24), Coffea canephora var. robusta genotypes (FRT64 and FRT05). The four maturation stages are: $S G$ small green grain, $L G$ large green grain, $Y G$ yellow grain, $R G$ red grain. The content is expressed in percentage of dry weight $(\%$ DW). Values are the mean of three repetitions \pm standard deviation
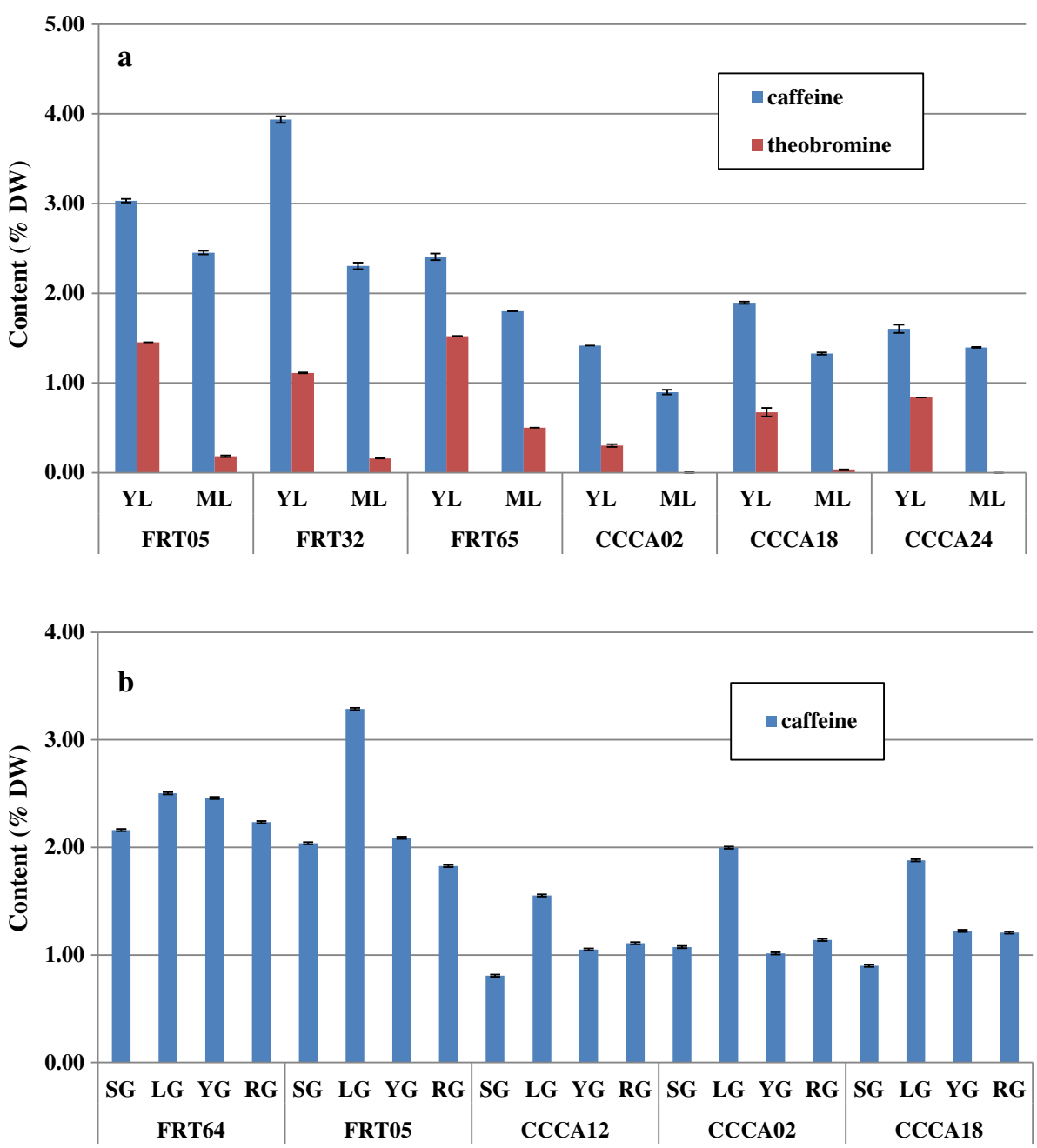

characterize the genes for Arabica. This strategy was highly successful since the complete set of genes involved in caffeine biosynthesis was amplified in Robusta and Arabica. In Robusta, three genes were identified: $C c X M T 1$ (JX978509), CcMXMT1 (JX978507) and CcDXMT (JX978506). In Arabica, six genes were identified: CaXMT1 (JX978514), CaXMT2 (JX978515), CaMXMT1 (JX978511), CaMXMT2 (JX978512), CaDXMT1 (KF678863) and CaDXMT2 (KJ577793) (Table 1). It is interesting to note that, based on the phylogenic analysis (Fig. 2), the different orthologues to CcXMT1, CcMXMT1 and $C c D X M T$ were clearly identified in $C$. arabica as CaXMT1, CaMXMT1 and CaDXMT2, respectively. This analysis suggests that these three genes are encoded by the sub-genome robusta in the Coffea arabica genome. Consequently, the additional genes CaXMT2, CaMXMT2, CaDXMT1 identified in Coffea arabica are certainly encoded by the sub-genome eugenioides.

These results are highly valuable since it is now possible to assign a specific gene to each sub-genome in Arabica, and thus to determine their respective importance for caffeine accumulation. This analysis gives a global overview of the different genes involved in the three final steps of caffeine synthesis in Arabica and Robusta species. The phylogenic analysis performed on the different sequences identified in the two Coffea species indicated that the encoded proteins are organized in three clusters, i.e., XMT, MXMT and DXMT (Fig. 2). Within the entire protein sequence, there are $16 \mathrm{key}$ amino acids that are highly informative for defining the different clusters (Table 2) and their corresponding enzymatic activities. This in-depth analysis lends considerable support to the previous results, showing key amino acids that define enzymatic activity (Ogawa et al. 2001; McCarthy and McCarthy 2007). For example, the role of $\mathrm{S}_{316}$ was shown to be crucial for xanthosine substrate specificity in XMT and $\mathrm{A}_{238}$ helped distinguish MXMT from DXMT (McCarthy and McCarthy 2007). In the perspective of acquiring coffee plants with reduced caffeine content, these different amino acids would be an interesting target for mutation using TILLING (Till et al. 2003; Kurowska et al. 2011) or TALEN (Beurdeley et al. 2013; Zhang et al. 2013) technology. After using the 

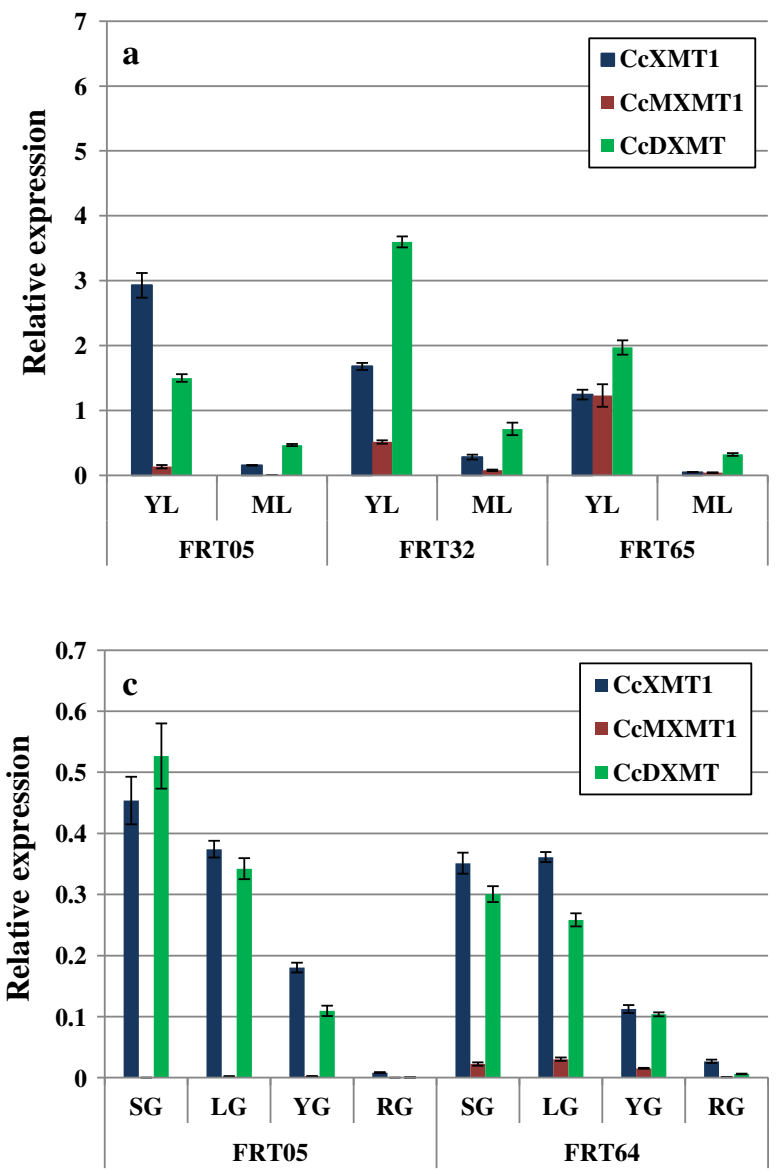

Fig. 4 Caffeine biosynthesis enzyme gene expression during leaf or grain development in Coffea arabica and Coffea canephora (Robusta), determined by q-RT-PCR. Transcript levels were analyzed during leaf development in young (YL) and mature leaves (ML) for different genotypes of Coffea canephora var. robusta (FRT05, FR32, FRT65, a) and Coffea arabica (CCCA 02, CCCA18, CCCA24, b). Transcript levels were analyzed during coffee bean maturation of different genotypes of Coffea canephora var. robusta (FRT64 and

strategy of "universal primers" to identify the different genes encoding the $\mathrm{N}$-methytransferase, surprisingly few additional genes were also amplified in Arabica and Robusta which encode MTL proteins, namely $N$-methyltransferase-like proteins (Uefuji et al. 2003). Previous results have shown that MTL protein are highly homologous with XMT, MXMT and DXMT enzymes, but do not participate in caffeine biosynthesis (Ogawa et al. 2001). A new phylogenic analysis was performed including the different MTL-encoding sequences identified in this work (Supplementary Fig. S2). The MTL proteins are specifically grouped in Cluster IV suggesting a stronger homology within the MTL proteins than with XMT, MXMT or DXMT proteins.

Caffeine metabolism in the Coffea species is set off by the degradation of purine nucleotides. Numerous publications investigated caffeine biosynthesis using radio-labeled
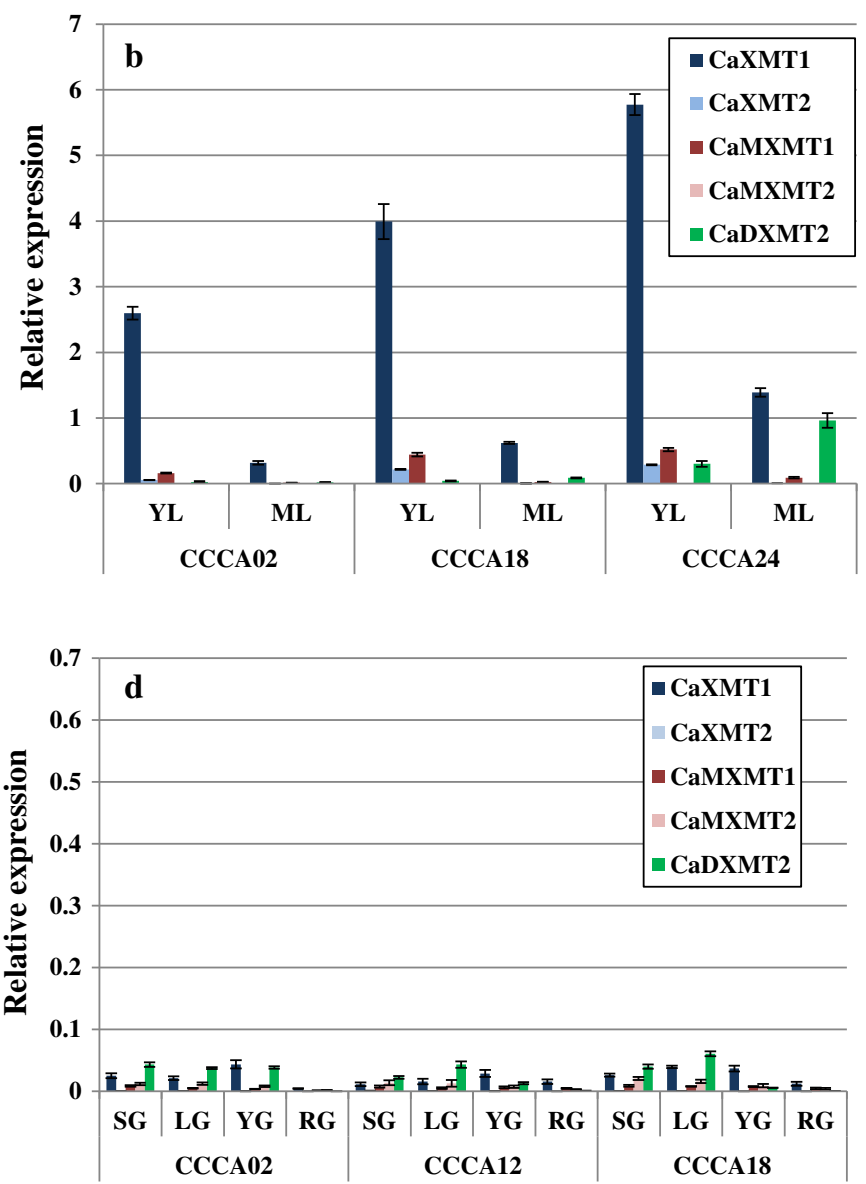

FRT05, c) and Coffea arabica (CCCA 02, CCCA12, CCCA18, d). The four maturation stages are: $S G$ small green grain, $L G$ large green grain, $Y G$ yellow grain, $R G$ red grain. The expression levels are determined by quantitative RT-PCR relative to the expression of the constitutively expressed $r p l 39$ gene in the same samples. In each case, values are the mean of three estimations \pm standard deviation. The same code color is used to characterize one gene in Robusta and its homologue in Arabica

tracers, metabolite profiling and enzymatic activities for leaf and fruit development (Suzuki and Waller 1984; Fujimori and Ashihara 1994; Zheng and Ashihara 2004; Campa et al. 2004; Koshiro et al. 2006). Active synthesis was detected in young leaves, bean and pericarp (Ashihara et al. 1996; Ashihara and Crozier 1999; De Castro and Marraccini 2006). In the present work, a large-scale analysis was performed to study the correlation between caffeine metabolism and gene expression during leaf and grain development in two Coffea species characterized by different caffeine accumulations. Previous gene expression analyses were performed by RT-PCR or classical Northern blot (Ogawa et al. 2001; Uefuji et al. 2003; Mizuno et al. 2003a, b). The high homology between the different sequences encoding the $N$-methyltransferases involved in caffeine metabolism reinforced our idea of screening for gene expression using quantitative RT-PCR, based on Taq- 
Man probe technology for ensuring the highest specificity and sensitivity for gene detection. To prevent any unspecific amplification, the primer/probe design for one specific gene was tested on the different sequences identified in this publication including MTL encoding genes. Only the primers/probe pairs that showed no unspecific amplification were used for further analysis. Unfortunately, none of the primers/probe pairs designed to analyze CaDXMT1 gene expression were specific or showed systematically cross hybridization with MTL encoding genes. Thusly, CaDXMT1 gene expression analysis was not thus analyzed using this technology. The second point to consider in q-RT-PCR analysis is the selection of accurate reference genes to be used for internal control for reliable data normalization. In keeping with previous data published in Coffea species, rpl39 (large ribosomal subunit 39) and $U B Q$ (ubiquitin-like protein) were used as reference gene (Privat et al. 2008; Salmona et al. 2008; Cruz et al. 2009; Lepelley et al. 2012). Similar results were obtained using both genes for internal control, suggesting a stable expression level throughout our experimental design.

For the first time, a global analysis was performed on leaf and grain throughout the development in various Robusta genotypes. Our data demonstrate that caffeine metabolism is highly active in young leaves (Fig. 3a) in Robusta, with caffeine levels reaching beyond $2 \%$ (DW). It is interesting to detect that caffeine accumulation seems to be highly dependant on $C c D X M T$ expression while theobromine is more closely related to the high accumulation of $C c X M T 1$ transcripts and to a lesser extend to CcMXMT1 since its expression is detected at a very low level in the three genotypes studied (Fig. 4a). During leaf development, theobromine content is drastically reduced, which is largely correlated to a lower $C c X M T 1$ transcripts accumulation. Caffeine is also less accumulated in mature leaves, which correlates to the reduction of $C c D X M T$ transcripts accumulation. Surprisingly, caffeine metabolism is lower in beans than during leaf development (Fig. 3a, b). These results are consistent with those obtained previously in leaves (Ashihara et al. 1996; Ashihara and Crozier 1999) or during grain development (Suzuki and Waller 1984; Ashihara and Suzuki 2004; Koshiro et al. 2006). Global transcriptional activity for the genes involved in caffeine metabolism is sharply reduced during bean development (10-20 times less) (Fig. 4a, c). Although, the gene expression pattern in Robusta is the same between endosperm and young leaves, transcript accumulation is significantly reduced for $C c X M T 1$ and $C c D X M T$ genes in the endosperm. CcXMT1 and CcDXMT transcripts are co-expressed, as it was observed in young leaves, with a decrease in accumulation during the last two stages of maturation. Despite the low accumulation of CcMXMT1 transcripts, the caffeine biosynthesis pathway is complete during bean maturation since the DXMT enzyme can methylate the 7-Methylxanthine and theobromine (Uefuji et al. 2003; McCarthy and McCarthy 2007).

In parallel, the same analysis was performed on leaf and grain in different arabica genotypes revealing great differences in caffeine metabolism depending on the organ and the species analyzed. In the different Arabica genotypes analyzed, caffeine and theobromine are less accumulated than in Robusta in leaves (Fig. 3a, b). At the same time, the gene expression pattern is markedly different, with a very high level of CaXMT1 expression, higher than its homologue in Robusta. CaXMT2 is over 10 times less expressed than CaXMT1 suggesting that CaXMT1 and CaXMT2 are controlled differentially at the transcriptional level. CaMXMT1 is less expressed than CcMXMT1; CaMXMT2 is almost undectectable in the different leaf tissues analyzed (Fig. 4b). These results strongly suggest that XMT1 and MXMT1 in both species drive theobromine accumulation, especially in young leaves. DXMT transcripts accumulation is remarkably different in Arabica. While $C c D X M T$ is strongly expressed and directly correlated with caffeine accumulation in leaves, CaDXMT2 transcripts are poorly accumulated partially explaining the reduced caffeine content in Arabica compared to Robusta. It is interesting to note that in Coffea arabica, by analyzing the genes involved in caffeine accumulation, the co-existence of the two sub-genomes has a clear impact on caffeine accumulation. The genes CaXMT1, CaMXMT1 and CaDXMT2 encoded by the Robusta sub-genome are expressed differently than their homologue in Robusta, drastically reducing caffeine accumulation. Secondly, the genes CaXMT2, CaMXMT2 and probably CaDXMT1 encoded by the sub-genome Eugenioides are expressed at very low level in Arabica as they certainly are in Coffea eugenioides, explaining the low caffeine metabolism (Ashihara and Crozier 1999; Campa et al. 2004; Ashihara 2006) in leaves (Fig. 4a, b). Nevertheless, the assumption made for CaDXMT1 transcript accumulation would need to be confirmed using RNA-seq technology, for example. With its single-base pair resolution, sensitivity, and replicability, this technology will be highly helpful in distinguishing $\mathrm{N}$-methyltransferase involved in caffeine metabolism and in determining the transcript accumulation in different organs. Gene expression for caffeine metabolism is especially low during Coffea arabica bean development; CaXMT1 and CaDXMT2 expression is drastically reduced compared to what was observed in leaves (Fig. 4b, d). This has a considerable impact on caffeine accumulation, reduced by $50 \%$ at the end of bean maturation. $\mathrm{Ca}$ $X M T 2$ and CaMXMT2 are poorly expressed during coffee bean development in Arabica, partially explaining why Coffea eugenioides also accumulates very low levels of caffeine in beans (Campa et al. 2004). In conclusion, 
XMT1 (CcXMT1 and CaXMT1) and DXMT (CcDXMT and CaDXMT2) genes play a major role in caffeine accumulation in leaf and bean in Arabica and Robusta. Previous gene expression in leaves or grain was performed and published using classical RT-PCR (Ogawa et al. 2001; Mizuno et al. 2003b; Koshiro et al. 2006). Similarly, major expression was identified for CaXMT1 and CaDXMT2 in Arabica even if quantitative analysis was not possible due to the limitations of the classical PCR. Despite the straightforward relationship between specific transcript accumulation and caffeine metabolism, the possibility of post-transcriptional regulation for these key genes XMT and DXMT needs to be considered even if it has not yet been proven.

A major goal of the coffee research programs is to identify and map quantitative trait loci (QTL) involved in cup quality and pathogen resistance and to use this data to apply MAS (Marker Assisted Selection). In parallel, two international sequencing initiatives have been launched to establish the genome sequence of the double haploid Coffea arabica and Coffea canephora var. robusta. In the near future, the resulting catalog of genetic and genomic information will help breeders integrate both analytical tools and release new coffee varieties with higher productivity, resistance and quality. It is probable that genomic locations of QTLs for sensory traits (bitterness) or caffeine content will coincide with the candidate genes characterized in this study, especially with the XMT1 and DXMT genes. The results presented here demonstrate that caffeine modulation content depends on the transcriptional activity monitoring differential expression patterns for the $X M T 1$ and DXMT genes, explaining the different levels of caffeine between Robusta and Arabica. Through this analysis, for the first time, it has been possible to investigate the impact of the co-existence of the two sub-genomes Robusta and Eugenioides in Coffea arabica on a specific metabolism. The lowest caffeine content in Arabica is due to reduced transcriptional activity controlling caffeine metabolism on a Robusta sub-genome associated with weak transcriptional activity from the eugenioides subgenome. The Robusta sub-genome has evolved in the Coffea arabica genetic background due to (1) possible evolution of its own transcriptional activity that controls caffeine metabolism or (2) negative impact from the eugenioides sub-genome, or both simultaneously. The data presented here points to interesting targets for modulating the final caffeine content of "green" coffee beans and hence for improving the quality of Robusta and Arabica coffee.

Author contribution IP and CP conceived and designed research. CP and GM conducted experiments. CP, SS, LM and ML contributed genomic and phylogenic analysis of the sequences. LB, SM and $\mathrm{JH}$ performed biochemical analysis. CP, ML, SS and IP analyzed the data. IP wrote the manuscript. All authors read and approved the manuscript.

Acknowledgments We are grateful to Alexandre de Kochko and Philippe Lashermes to have provided samples from the doubledhaploid Coffea canephora and Coffea arabica, respectively. We wish to thank Victoria Berry for generating certain RNA/cDNA samples used in the q-RT-PCR experiments. We wish to thank Anne Lussiez for the early work done on gene identification involved in the caffeine pathway.

Open Access This article is distributed under the terms of the Creative Commons Attribution License which permits any use, distribution, and reproduction in any medium, provided the original author(s) and the source are credited.

\section{References}

Altschul SF, Gish W, Miller W, Myers EW, Lipman DJ (1990) Basic local alignment search tool. J Mol Biol 215:403-410

Ashihara H (2006) Metabolism of alkaloids in coffee plants. Braz J Plant Physiol 18:1-8

Ashihara H, Crozier A (1999) Biosynthesis and catabolism of caffeine in low-caffeine-containing species of coffea. J Agric Food Chem 47:3425-3431

Ashihara H, Suzuki T (2004) Distribution and biosynthesis of caffeine in plants. Front Biosci 9:1864-1876

Ashihara H, Monteiro AM, Gillies FM, Crozier A (1996) Biosynthesis of caffeine in leaves of coffee. Plant Physiol 111:747-753

Ashihara H, Sano H, Crozier A (2008) Caffeine and related purine alkaloids: biosynthesis, catabolism, function and genetic engineering. Phytochemistry 69:841-856

Bargel H, Neinhuis C (2005) Tomato (Lycopersicon esculentum Mill.) fruit growth and ripening as related to the biomechanical properties of fruit skin and isolated cuticle. J Exp Bot 56:1049-1060

Beurdeley M, Bietz F, Li J, Thomas S, Stoddard T, Juillerat A, Zhang F, Voytas DF, Duchateau P, Silva GH (2013) Compact designer TALENs for efficient genome engineering. Nat Commun 4:1762

Brandt BW, Feenstra KA, Heringa J (2010) Multi-Harmony: detecting functional specificity from sequence alignment. Nucleic Acids Res 38:W35-W40

Campa C, Doulbeau S, Dussert S, Hamon S, Noirot M (2004) Diversity in bean caffeine content among wild coffea species: evidence of a discontinuous distribution. Food Chem 91:633-637

Campa C, Doulbeau S, Dussert S, Hamon S, Noirot M (2005) Qualitative relationship between caffeine and chlorogenic acid contents among wild coffea species. Food Chem 93:135-139

Cruz F, Kalaoun S, Nobile P, Colombo C, Almeida J, Barros LMG, Romano E, Grossi-de-Sa MF, Vaslin M, Alves-Ferreira M (2009) Evaluation of coffee reference genes for relative expression studies by quantitative real-time RT-PCR. Mol Breed 23:607-616

De Castro RD, Marraccini P (2006) Cytology, biochemistry and molecular changes during coffee fruit development. Braz J Plant Physiol 18:175-199

Fujimori N, Ashihara H (1994) Biosynthesis of theobromine and caffeine in developing leaves of Coffea arabica. Phytochemistry 36:1359-1361

Koshiro Y, Zheng XQ, Wang ML, Nagai C, Ashihara H (2006) Changes in content and biosynthetic activity of caffeine and 
trigonelline during growth and ripening of Coffea arabica and Coffea canephora fruits. Plant Sci 171:242-250

Kurowska M, Daszkowska-Golec A, Gruszka D, Marzec M, Szurman M, Szarejko I, Maluszynski M (2011) TILLING: a shortcut in functional genomics. J Appl Genet 52:371-390

Ky CL, Louarn J, Dussert S, Guyot B, Hamon S, Noirot M (2001) Caffeine, trigonelline, chlorogenic acids and sucrose diversity in wild Coffea arabica L. and C. canephora P. accessions. Food Chem 75:223-230

Ky CL, Barre P, Noirot M (2013) Genetic investigations on the caffeine and chlorogenic acid relationship in an interspecific cross between Coffea liberica dewevrei and C. pseudozanguebariae. Tree Genet Genomes 9:1043-1049

Lashermes P, Combes MC, Robert J, Trouslot P, D'Hont A, Anthony F, Charrier A (1999) Molecular characterisation and origin of the Coffea arabica L. genome. Mol Gen Genet 261:259-266

Lepelley M, Simkin A, Cheminade G, Caillet V, Tremillon N, McCarthy J (2007) Chlorogenic acid synthesis in coffee: An analysis of CGA content and real-time RT-PCR expression of HCT, HQT, C3H1 and CCoAOMT1 genes during grain development in C. canephora. Plant Sci 172:978-996

Lepelley M, Mahesh V, McCarthy J, Rigoreau M, Crouzillat D, Chabrillange N, de Kochko A, Campa C (2012) Characterization, high-resolution mapping and differential expression of three homologous $P A L$ genes in Coffea canephora Pierre (Rubiaceae). Planta 236:313-326

Leroy T, Ribeyre F, Bertrand B, Charmetant P, Dufour M, Montagnon C, Marraccini P, Pot D (2006) Genetics of coffee quality. Braz J Plant Physiol 18:229-242

McCarthy AA, McCarthy JG (2007) The structure of two $\mathrm{N}$ methyltransferases from the caffeine biosynthetic pathway. Plant Physiol 144:879-889

McCarthy AA, Biget L, Lin C, Petiard V, Tanksley SD, McCarthy JG (2007) Cloning, expression, crystallization and preliminary $\mathrm{X}$-ray analysis of the XMT and DXMT $N$-methyltransferases from Coffea canephora (robusta). Acta Crystallogr, Sect F: Struct Biol Cryst Commun 63:304-307

Mizuno K, Kato M, Irino F, Yoneyama N, Fujimura T, Ashihara H (2003a) The first committed step reaction of caffeine biosynthesis: 7-methylxanthosine synthase is closely homologous to caffeine synthases in coffee (Coffea arabica L.). FEBS Lett 547:56-60

Mizuno K, Okuda A, Kato M, Yoneyama N, Tanaka H, Ashihara H, Fujimura T (2003b) Isolation of a new dual-functional caffeine synthase gene encoding an enzyme for the conversion of 7-methylxanthine to caffeine from coffee (Coffea arabica L.). FEBS Lett 534:75-81

Montagnon C, Guyot B, Cilas C, Leroy T (1998) Genetic parameters of several biochemical compounds from green coffee, Coffea canephora. Plant Breed 117:576-578

Ogawa M, Herai Y, Koizumi N, Kusano T, Sano H (2001) 7-Methylxanthine methyltransferase of coffee plants. Gene isolation and enzymatic properties. J Biol Chem 276:8213-8218

Privat I, Foucrier S, Prins A, Epalle T, Eychenne M, Kandalaft L, Caillet V, Lin C, Tanksley S, Foyer C, McCarthy J (2008)
Differential regulation of grain sucrose accumulation and metabolism in Coffea arabica (Arabica) and Coffea canephora (Robusta) revealed through gene expression and enzyme activity analysis. New Phytol 178:781-797

Rogers WJ, Michaux S, Bastin M, Bucheli P (1999) Changes to the content of sugars, sugar alcohols, myo-inositol, carboxylic acids and inorganic anions in developping grains from different varieties of Robusta (Coffea canephora) and Arabica (C. arabica) coffee. Plant Sci 149:115-123

Salmona J, Dussert S, Descroix F, de Kochko A, Bertrand B, Joet T (2008) Deciphering transcriptional networks that govern Coffea arabica seed development using combined cDNA array and realtime RT-PCR approaches. Plant Mol Biol 66:105-124

Simkin AJ, Qian T, Caillet V, Michoux F, Ben Amor M, Lin C, Tanksley S, McCarthy J (2006) Oleosin gene family of Coffea canephora: quantitative expression analysis of five oleosin genes in developing and germinating coffee grain. J Plant Physiol 163:691-708

Suzuki T, Waller GR (1984) Biosynthesis and biodegradation of caffeine, theobromine, and theophylline in Coffea arabica L. fruits. J Agric Food Chem 32:845-848

Tamura K, Peterson D, Peterson N, Stecher G, Nei M, Kumar S (2011) MEGA5: molecular evolutionary genetics analysis using maximum likelihood, evolutionary distance, and maximum parsimony methods. Mol Biol Evol 28:2731-2739

Thompson JD, Higgins DG, Gibson TJ (1994) CLUSTAL W: improving the sensitivity of progressive multiple sequence alignment through sequence weighting, position-specific gap penalties and weight matrix choice. Nucleic Acids Res 22:4673-4680

Till BJ, Colbert T, Tompa R, Enns LC, Codomo CA, Johnson JE, Reynolds SH, Henikoff JG, Greene EA, Steine MN, Comai L, Henikoff S (2003) High-throughput TILLING for functional genomics. Methods Mol Biol 236:205-220

Uefuji H, Ogita S, Yamaguchi Y, Koizumi N, Sano H (2003) Molecular cloning and functional characterization of three distinct $\mathrm{N}$-methyltransferases involved in the caffeine biosynthetic pathway in coffee plants. Plant Physiol 132:372-380

Uefuji H, Tatsumi Y, Morimoto M, Kaothien-Nakayama P, Ogita S, Sano H (2005) Caffeine production in tobacco plants by simultaneous expression of three coffee $N$-methyltransferases and its potential as a pest repellant. Plant Mol Biol 59:221-227

Waterhouse AM, Procter JB, Martin DM, Clamp M, Barton GJ (2009) Jalview version 2-a multiple sequence alignment editor and analysis workbench. Bioinformatics 25:1189-1191

Wintgens J (2004) Coffee: growing, processing, sustainable production: a guidebook for growers, processors, traders and researchers. Wiley-VCH Verlag GmbH, Weinheim, pp 1-976

Zhang Y, Zhang F, Li X, Baller JA, Qi Y, Starker CG, Bogdanove AJ, Voytas DF (2013) Transcription activator-like effector nucleases enable efficient plant genome engineering. Plant Physiol 161:20-27

Zheng XQ, Ashihara H (2004) Distribution, biosynthesis and function of purine and pyridine alkaloids in Coffea arabica seedlings. Plant Sci 166:807-813 